\title{
Effect of $n-3$ long-chain polyunsaturated fatty acid intake on the eicosanoid profile in individuals with obesity and overweight: a systematic review and meta-analysis of clinical trials
}

\author{
Guilherme R. B. Schweitzer ${ }^{1}$, Isabela N. M. S. Rios ${ }^{1}$, Vivian S. S. Gonçalves ${ }^{2}$, Kelly G. Magalhães ${ }^{3}$ (1) and \\ Nathalia Pizato $^{1 *}$ (1) \\ ${ }^{1}$ Graduate Program in Human Nutrition, Department of Nutrition, University of Brasilia, Brasilia, Brazil \\ ${ }^{2}$ Graduate Program in Public Health, University of Brasilia, Brasilia, Brazil \\ ${ }^{3}$ Laboratory of Immunology and Inflammation, Department of Cellular Biology, Institute of Biology, University of Brasilia, Brasília, Brazil
}

(Received 11 June 2021 - Accepted 25 June 2021)

Journal of Nutritional Science (2021), vol. 10, e53, page 1 of 15

doi:10.1017/jns.2021.46

Abstract

Dietary $n-3$ polyunsaturated fatty acids (PUFAs) present beneficial effects on counteracting inflammation status, displaying a critical anti-inflammatory role and maintaining physiological homeostasis in obesity. The primary objective of this systematic review was to evaluate the effect of $n$-3 PUFAs intake on the eicosanoid profile of people with obesity and overweight. The search strategy on Embase, Scopus, PubMed, Web of Science, Cochrane Library, Google Scholar and ProQuest was undertaken until November 2019 and updated January 2021. The effect size of $n$-3 PUFAs on prostaglandins was estimated by Glass's, type 1 in a random-effect model for the meta-analysis. Seven clinical trials met the eligible criteria and a total of 610 subjects were included in this systematic review, and four of seven studies were included in meta-analysis. The intake of $n-3$ PUFAs promoted an overall reduction in serum proinflammatory eicosanoids. Additionally, $n$-3 PUFAs intake significantly decreased the arachidonic acid COX-derived PG eicosanoid group levels (Glass's $\Delta-0 \cdot 35$; CI $\left.-0 \cdot 62,-0 \cdot 07, I^{2} 31 \cdot 48\right)$. Subgroup analyses showed a higher effect on periods up to 8 weeks (Glass's $\Delta-0 \cdot 51 ;$ CI $\left.-0 \cdot 76,-0 \cdot 27\right)$ and doses higher than $0.5 \mathrm{~g}$ of $n$-3 PUFAs (Glass's $\Delta-0 \cdot 46$; CI $-0 \cdot 72,-0 \cdot 27$ ). Dietary $n-3$ PUFAs intake contributes to reduce pro-inflammatory eicosanoids of people with obesity and overweight. Subgroup's analysis showed that $n$ - 3 PUFAs can reduce the overall arachidonic acid COX-derived PG when adequate dose and period are matched.

Key words: Eicosanoids: Inflammation: $n$-3 PUFA: Obesity: Systematic review

\section{Introduction}

Obesity is one of the most prevalent chronic conditions presently seen in western society ${ }^{(1)}$ and it leads to increase activation of inflammatory signalling, characterised by a rise in eicosanoid levels ${ }^{(2)}$. In individuals with obesity, visceral adipose tissue presents a dysfunctional phenotype when compared with lean individuals' tissue. Excessive adipose tissue accumulation can potentially modify phospholipase A2 (PLA2), cyclooxygenase 2 (COX-2) and 5-lipooxygenase (5-LOX) activity, thereby increasing bioactive eicosanoid mediators such as prostaglandins (PG) and leukotrienes $(\mathrm{LT})^{(3,4)}$.
This metabolic modification seems to determine a state of chronic low-grade inflammation, which is recognised as a critical factor for the establishment and progression of metabolic dysfunction associated with obesity ${ }^{(5)}$. These obesity-related comorbidities are closely linked to the presence of a persistent activation of pro-inflammatory signalling pathways in adipose tissue, which severely disrupts key metabolic checkpoints in this tissue ${ }^{(5)}$. The chronic pro-inflammatory eicosanoid synthesis is recognised as a key factor in the development of insulin resistance $^{(6)}$, tissue inflammation ${ }^{(7)}$, renal injury ${ }^{(8)}$ and increased cardiovascular maladaptations ${ }^{(9)}$. In addition to the

*Corresponding author: Nathalia Pizato, email pizatonat@unb.br 
heightened production of inflammatory mediators, obese adipose tissue shows an intrinsic inability to resolve uncontrolled inflammation and to restore tissue homeostasis and functionality $^{(10)}$. Titos et al. identified a group of genes associated with the inflammatory process that was differentially modulated in people with obesity, and COX-2 was also significantly up-regulated in their adipose tissue ${ }^{(11)}$. In obesity, the main problem is the imbalances in the complex network of eicosanoids synthesis, resulting from the chronicity of the inflammatory response.

Inflammation and dietary fat, especially $n-6 / n-3$ polyunsaturated fatty acids (PUFAs) ratio, have a tight correlation; ${ }^{(12)}$ therefore, the composition of the cell membrane influences eicosanoid metabolism. There is enzymatic competition by the use of these PUFAs as substrates for inflammatory mediators synthesis ${ }^{(2)}$, and the higher $n-6 / n-3$ PUFA ratio intake is directly associated with augmentation in COX and LOX-derived pro-inflammatory eicosanoids ${ }^{(13,14)}$ such as prostaglandin E2 (PGE2), leukotriene B4 (LTB4) and thromboxane A2 (TXA2), derived from $n-6$ fatty acids such as arachidonic acid $(\mathrm{AA})^{(15)}$. Bowers et al. demonstrated in the breast tissue of women with obesity a greater macrophage COX-2 expression and more PGE2 production ${ }^{(16)}$. In individual with obesity and diabetes mellitus type 2 , the production of pro-inflammatory AA metabolites, including PGE2, leukotrienes (LT) and HETEs, was related to insulin resistance, and PGF2 $\alpha$ was linked to hepatic gluconeogenesis, a major driver of fasting hyperglycaemia ${ }^{(17)}$.

Conversely, higher plasma levels of $n-3$ PUFA are associated with reduced obesity risk ${ }^{(18)}$. since the intake of $n-3$ eicosapentaenoic acid (EPA) has the capability to modulate eicosanoid synthesis profile in COX and LOX-dependent pathways, generating the 3-series PG and TX, and 5-series LT and lipoxins (LX), which are less bioactive eicosanoids than the ones generated by $\mathrm{AA}^{(19)} \cdot n-3$ docosaexaenoic acid (DHA) intake is associated with docosanoid synthesis, a similar yet different category of molecules that include resolvins, protectins, neuroprotectins and maresins, commonly referred to as specialised pro-resolvin mediators $(\mathrm{SPMs})^{(20,21)}$. Because of enzymatic competition, a higher EPA and DHA consumption might shift bioactive eicosanoid synthesis from AA metabolites (pro-inflammatory activity) to EPA and DHA metabolites (less pro-inflammatory activity and even modestly anti-inflammatory activity) ${ }^{(22)}$. Besides COX and LOX enzyme pathway, cytochrome P450 enzyme family also synthesise eicosanoid mediators as 5-HEPE (EPA-derived) and 5-HETE (AA-derived) contributing to modulate inflammation response ${ }^{(23,24)}$. These findings reinforce and strengthen the importance of COX and LOX mediator's activation in the development of obesity-related complications.

Significant modifications of inflammatory signalling and serum eicosanoid profile have been seen during intervention with EPA and DHA fatty acids ${ }^{(25,26)}$, suggesting a protective effect of $n-3$ PUFA. A recent previous study ${ }^{(27)}$ showed that marine-derived $n-3$ PUFA presented a beneficial effect on reducing major pro-inflammatory eicosanoids serum values in unhealthy subjects. However, to the best of our knowledge, there has been no study conducted to summarise the available evidence of the effects of $n-3$ PUFA intake on eicosanoid, markers of inflammation, of individuals with obesity and overweight without chronic diseases. Therefore, the aim of the present study was to evaluate the effects of $n$ - 3 PUFA intake on a variety of eicosanoids on adults with obesity and overweight, through a systematic review and meta-analysis of controlled trials.

\section{Materials and methods}

The current systematic review and meta-analysis was conducted as recommended by the state-of-the-art method Preferred Reporting Items for Systematic Reviews and Meta-analyses (PRISMA - Appendix 1) ${ }^{(28)}$, and the protocol was registered in Prospective Register of Systematic Reviews (PROSPERO - CRD42020153362).

\section{Information sources and search strategies}

A comprehensive search was executed in the following databases: PubMed, Cochrane library, Embase, Scopus and Web of Science, and grey literature (Google Scholar and ProQuest). Publications up to 7 November 2019 were examined, and updated on 25 January 2021.

The search strategy was reviewed by an investigator with experience in systematic reviews in accordance with the Peer Review of Electronic Search Strategies (PRESS) checklist criteria $^{(28)}$. The following strategy was adapted for databases: ('Morbid obesity' OR 'Severe obesity' OR 'Abdominal obesity' OR 'Central obesity' OR 'Visceral obesity' OR 'Obese men' OR ‘Obese women' OR ‘Overweight' OR ‘Overweight men' OR 'Overweight women' OR 'Excess weight' OR 'obese' OR ‘obesity' OR 'Fat accumulation' OR 'fatness' OR 'body fatness' for population main characteristic and combined with intervention keywords ' $N 3$ fatty acids' OR ' $n-3$ Fatty Acids' OR ' $n 3$ Fatty Acids' OR ' $n 3$ Fatty Acids' 'W3 fatty acids' OR 'w-3 fatty acids' OR 'w 3 fatty acids' OR 'N3 Polyunsaturated Fatty Acid' OR ' $n-3$ Polyunsaturated Fatty Acid' OR ' $n 3$ Polyunsaturated Fatty Acid' OR ' $n 3$ Polyunsaturated Fatty Acid' OR ' $n-3$ PUFA' OR ' $N 3$ PUFA' OR ' $N 3$ PUFA' OR ' $N-3$ oils' $O R$ ' $N 3$ oils' $O R$ 'N 3 oils' OR 'Omega 3 Fatty Acids' OR 'Eicosapentanoic Acid' OR 'omega 3 Eicosapentaenoic Acid' OR 'omega-3-Eicosapentaenoic Acid' OR 'Timnodonic Acid' OR 'Docosahexenoic Acid' OR 'omega 3 Docosahexenoic Acid' OR 'Docosahexaenoate' OR ‘alpha Linolenic Acid' OR 'Linolenate' OR 'Linolenic Acid' OR 'EPA and DHA supplementation' OR EPA OR DHA OR ‘omega 3' OR ‘omega-3' OR 'fish oil' OR 'arachidonic acid' OR 'arachidonate') AND ('eicosatetraenoic acid' OR eicosanoid OR Icosanoid OR Prostanoid OR Lipoxin OR Prostaglandin OR Thromboxane OR Leukotriene OR 'hydroxyeicosatetraenoic acid' OR 'Isoprostane' OR 'dinoprostone'). The Google search was limited to the first 200 most relevant articles. No filters on language, publication date or status were applied to the results found in each database. More information about search strategies is provided in Appendix 2.

A consultation was carried out on the ClinicalTrials.gov Database portal (U.S. National Library of Medicine) in order 
to verify if there was any on-going or non-published article that could be included in our systematic review. Reference lists of included records were manually reviewed to identify potential studies not retrieved from databases.

Study selection was undertaken independently by two reviewers (G. S. and I. R.). The duplicates were removed, and the screening procedure was applied using Rayyan software ${ }^{(29)}$. Both authors (G. S. and I. R.) independently assessed the full text of preselected studies for eligibility. Disagreements were resolved by consensus and another author (V. S. S. G.) if required.

\section{Eligibility criteria}

Clinical trials studies that were conducted on adults with obesity and overweight (more than 18 years old and less than 65 years old) were eligible. The intervention criteria were $n-3$ PUFA intake either through oral supplements or foods.

Exclusion criteria were as follows: (1) studies with subjects that underwent bariatric/metabolic surgery; (2) consensus, management, reviews, letters, conference abstracts, editorials; (3) studies evaluating subjects with inflammatory and chronic diseases; (4) use of non-steroidal anti-inflammatory drugs; and (5) absent eicosanoid measurement or measurements other than serum or plasma eicosanoids.

\section{Data extraction}

Data were extracted undertaken independently by two investigators (G. S. and I. R.), with discrepancies resolved through group discussion. The extracted information was categorised as follows: (1) Author, Year of publication, and Country; (2) Study design; (3) Study Period; (4) n-3 PUFA source; (5) Study Protocol; (6) Food or supplement adherence protocol; (7) Age; (8) Baseline BMI; (9) Baseline and Post-Intervention serum eicosanoids markers; and (10) Main results.

\section{Risk of bias in individual studies}

The risk of bias of included articles was performed according to the Joanna Briggs Institute Critical Appraisal Tools. A 9-question checklist ('Checklist of quasi-experimental studies') was used to assess risk of bias in non-randomised clinical trials and a 13-question checklist ('Checklist of randomised controlled trials') in order to evaluate randomised clinical trials. The tool consisted of 9 or 13 questions, always answered as 'yes', 'no', 'unclear' or 'not applicable'. In the present study, the risk of bias was considered low when all items were answered as 'yes'. If any item was classified as 'no', a high risk of bias was expected. The risk of bias of each included study was independently certified by two reviewers (G. S. and I. R.).

\section{Summary measures and synthesis of results}

The primary outcomes were the identified measures of association between the $n$-3 PUFA intake and serum eicosanoids concentration for qualitative analysis.

Due to available data collected regarding the primary outcomes, we were able to conduct a meta-analysis to investigate the effect size of $n-3$ PUFA intake on PG concentrations. For this, we built a random-effect model using the restricted maximumlikelihood (REML) method ${ }^{(30)}$. The random-effect meta-analysis approach incorporates an assumption that the different studies are estimating different, yet related, intervention effects ${ }^{(31,32)}$.

The difference between the parameters investigated from baseline to end point was estimated by Glass's $\Delta$ type 1 with its respective $95 \% \mathrm{CI}^{(33)}$. The Glass's $\Delta$ is suitable for studies whose comparison with a control group is not possible ${ }^{(34)}$. In studies with more than one intervention group, the highest $n$ - 3 PUFA dose group was considered for analysis. In the study that evaluated salmon and cod intake, only the salmon group was included for analysis since salmon provided seven times more $n-3$ PUFA when compared to cod. For the study with a crossover design, mean changes between the levels of markers at the end of two intervention periods were used according to Cochrane Handbook ${ }^{(35)}$.

Heterogeneity of treatment effects between studies was tested using the $\chi^{2}$ method $(P<0 \cdot 10)$ and its magnitude using $I^{2}$. When $I^{2}$ was less than $40 \%$, it was not considered important, according to Cochrane's collaboration recommendation ${ }^{(36)}$. In order to investigate parameters influencing heterogeneity, we performed subgroup analyses, considering the following strata: doses of $n-3$ (higher or lower than $0.5 \mathrm{~g}$ EPA + DHA n-3 PUFA as recommended by ISSFAL for the general adult population ${ }^{(37)}$ ); source of administration of $n-3$ (food or oral supplement) and intervention time (more than 8 weeks or up to 8 weeks). Due to the small number of studies included in the meta-analysis, it was not possible to perform meta-regression and analysis of publication bias ${ }^{(32)}$.

\section{Results}

\section{Selection of relevant studies}

The initial search identified a total of 3641 articles from 7 databases, and after removing duplicates, 39 potential studies met the eligibility assessment and complete full-text reading. Thirty-two articles were excluded and the reasons are presented in Appendix 3. At the end, seven articles ${ }^{(38-44)}$ were selected for this systematic review. After selection, the full reference list of each article was checked in order to identify possible additions and no article was potentially eligible. In addition, the Clinicaltrials.gov registers were consulted and no protocol associated with eligible articles has been identified. A flow diagram of the screening process is shown in Fig. 1.

Seven studies were selected for qualitative analysis. Of these, three studies were excluded from meta-analysis because the required data were not available. One study did not analyse PG, one was excluded because only provided figures without quantitative information, and one present insufficient information (no data at the baseline in control groups). We contacted the authors for data but received no replies.

\section{Characteristics of included studies}

The characteristics of the studies included are summarised in Table 1. Seven clinical trials included 610 individuals with obesity and/or overweight published between $2010^{(44)}$ and $2019^{(38)}$ (Table 1). Among them, six studies ${ }^{(39-44)}$ are 


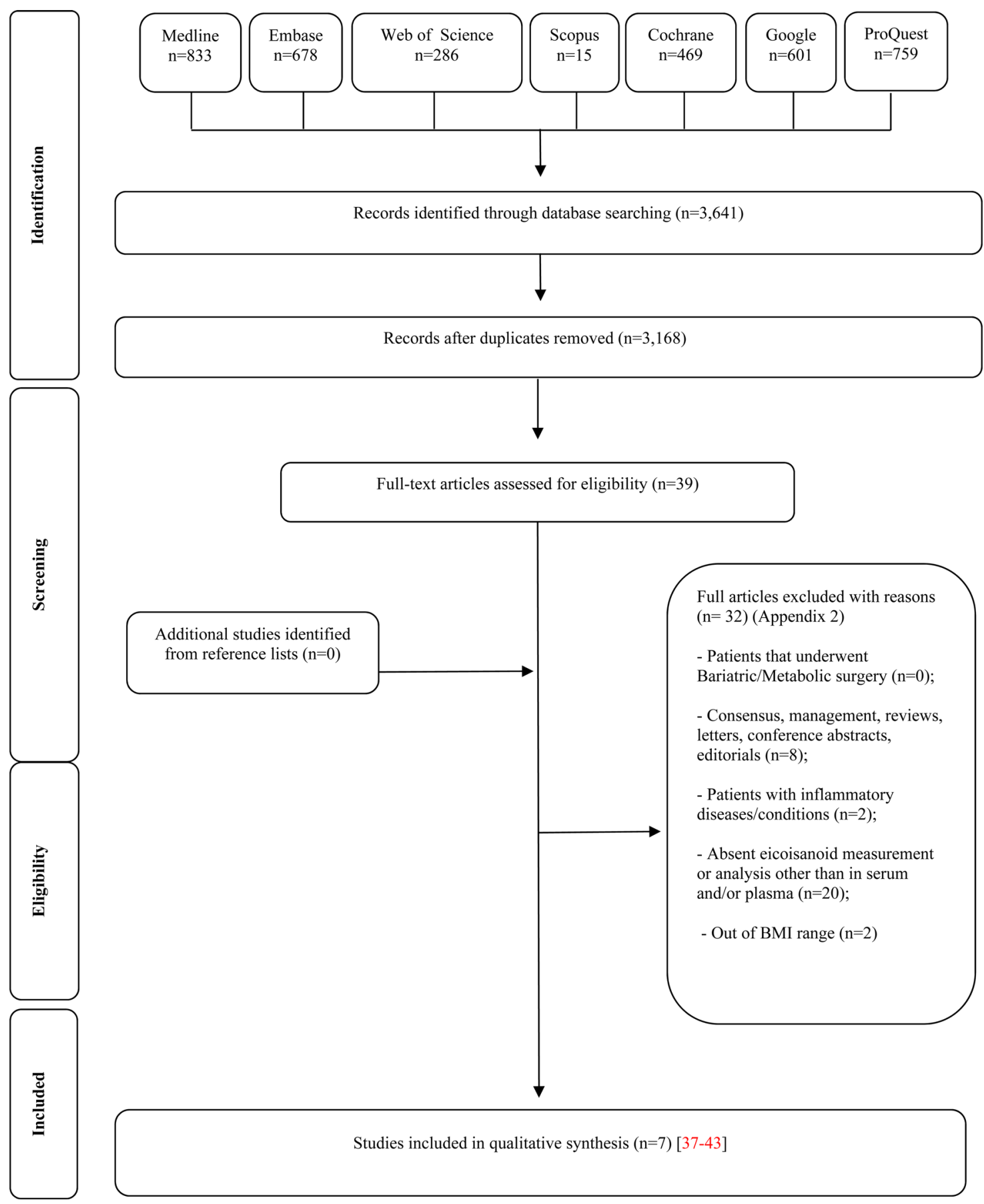

Fig. 1. Flow diagram of studies evaluated in the review (adapted from PRISMA).

randomised trials and only one study ${ }^{(38)}$ is a non-randomised trial. The studies were conducted in different countries, including Spain ${ }^{(38,40)}$, Germany ${ }^{(39)}$, Denmark ${ }^{(41)}$ and Poland ${ }^{(43)}$. Ramel et al. was a multicenter study that included Spain, Iceland and Ireland ${ }^{(44)}$.

Generally, participants were middle-aged and overweight. The lower baseline BMI was $25 \cdot 91 \pm 3 \cdot 67^{(39)}$ and the higher BMI was $34 \cdot 4 \pm 2 \cdot 69^{(43)}$. The mean age ranged from $31 \pm 5 \cdot 9$ years old $^{(44)}$ to $61 \cdot 82 \pm 7 \cdot 13^{(39)}$. The majority of studies analysed both men and women ${ }^{(39-42,44)}$, except by Celada et al. and Polus et al., which included only men and only women, respectively. The intervention period ranged from $4^{(38)}$ to 24 weeks ${ }^{(40)}$.
The $n-3$ PUFA content of the protocol interventions was provided by oral oil supplements ${ }^{(40-44)}$ or by food such as $n-3$ enriched frankfurters and patés ${ }^{(38)}, n-3$ enriched yoghurt $^{(39)}$ and salmon ${ }^{(44)} \cdot n-3$ enriched food was provided as a mixture of different $n-3$ long-chain PUFA family, including EPA, DHA, DPA (docosapentaenoic acid) ${ }^{(38)}$ and ALA (alpha-linolenic acid) ${ }^{(39)}$. One study supplemented individuals using exclusively DHA fatty acid capsules ${ }^{(40)}$ and three other studies supplemented with EPA plus DHA capsules ${ }^{(41-43)}$. Only one study presented the total fat amount and did not mention which specific fatty acids were included in the intervention protocol ${ }^{(44)}$. The control group were supplemented 
Table 1. Summary of descriptive characteristics and outcomes of interest of the included studies $(n 7)$

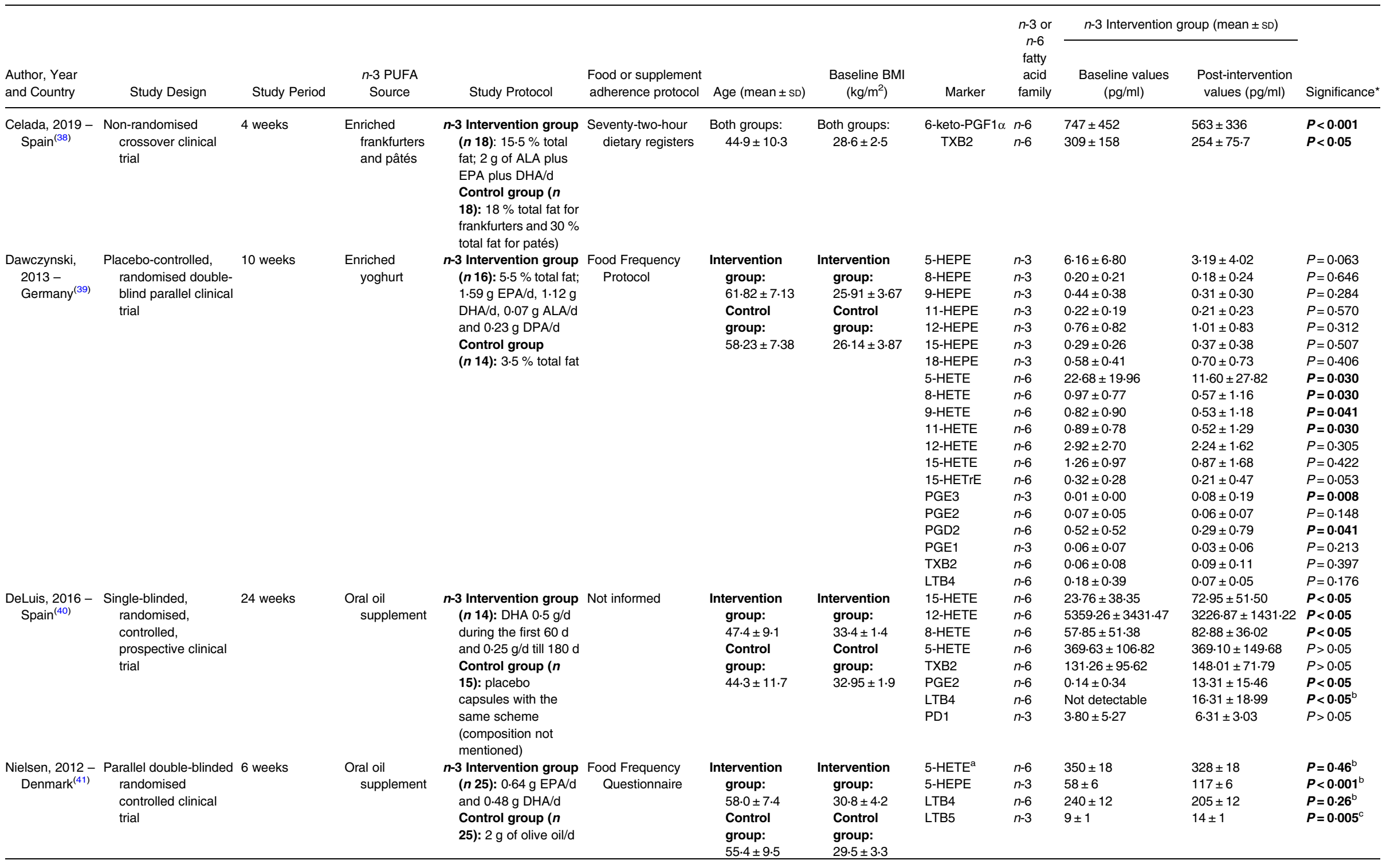




\begin{tabular}{|c|c|c|c|c|c|c|c|c|c|c|c|c|}
\hline \multirow[b]{2}{*}{$\begin{array}{l}\text { Author, Year } \\
\text { and Country }\end{array}$} & \multirow[b]{2}{*}{ Study Design } & \multirow[b]{2}{*}{ Study Period } & \multirow[b]{2}{*}{$\begin{array}{l}n \text {-3 PUFA } \\
\text { Source }\end{array}$} & \multirow[b]{2}{*}{ Study Protocol } & \multirow[b]{2}{*}{$\begin{array}{l}\text { Food or supplement } \\
\text { adherence protocol }\end{array}$} & \multirow[b]{2}{*}{ Age $($ mean $\pm \mathrm{SD})$} & \multirow[b]{2}{*}{$\begin{array}{c}\text { Baseline BMI } \\
\left(\mathrm{kg} / \mathrm{m}^{2}\right)\end{array}$} & \multirow[b]{2}{*}{ Marker } & \multirow{2}{*}{$\begin{array}{c}n-3 \text { or } \\
n-6 \\
\text { fatty } \\
\text { acid } \\
\text { family }\end{array}$} & \multicolumn{2}{|c|}{$n-3$ Intervention group $($ mean $\pm \mathrm{SD}$ ) } & \multirow[b]{2}{*}{ Significance* } \\
\hline & & & & & & & & & & $\begin{array}{l}\text { Baseline values } \\
(\mathrm{pg} / \mathrm{ml})\end{array}$ & $\begin{array}{l}\text { Post-intervention } \\
\text { values (pg/ml) }\end{array}$ & \\
\hline $\begin{array}{l}\text { O'Sullivan, } \\
2014- \\
\text { United } \\
\text { States }^{(42)}\end{array}$ & $\begin{array}{l}\text { Double-blind, placebo } \\
\text { controlled } \\
\text { randomised clinical } \\
\text { trial }\end{array}$ & 6 weeks & $\begin{array}{l}\text { Oral oil } \\
\text { supplement }\end{array}$ & 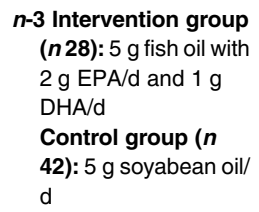 & $\begin{array}{l}\text { Food Frequency } \\
\text { Questionnaires }\end{array}$ & $\begin{array}{l}\text { Intervention } \\
\text { group: } \\
37 \cdot 2 \pm 12 \\
\text { Control } \\
\text { group: } \\
34 \cdot 1 \pm 12\end{array}$ & $\begin{array}{l}\text { Intervention } \\
\text { group: } \\
27 \cdot 0 \pm 4 \cdot 3 \\
\text { Control } \\
\text { group: } \\
27 \cdot 7 \pm 4 \cdot 6\end{array}$ & $\begin{array}{l}\text { 5-HEPE } \\
\text { LTB4 }^{\mathrm{d}}\end{array}$ & $\begin{array}{l}n-3 \\
n-6\end{array}$ & $\begin{array}{l}\text { Slope }=11, r^{2} 0.55(r \\
\text { Slope }=-2 \cdot 0, r^{2} 0.25\end{array}$ & $\begin{array}{l}28) \\
(n 29)\end{array}$ & $\begin{array}{l}P<0.0001 \\
P=0.005\end{array}$ \\
\hline $\begin{array}{l}\text { Polus, } 2016 \text { - } \\
\text { Poland }^{(43)}\end{array}$ & $\begin{array}{l}\text { Randomised } \\
\text { placebo-controlled } \\
\text { double-blind clinical } \\
\text { trial }\end{array}$ & 12 weeks & $\begin{array}{l}\text { Oral oil } \\
\text { supplement }\end{array}$ & $\begin{array}{l}n-3 \text { Intervention group } \\
(n \text { 24): } 1.29 \mathrm{~g} \mathrm{DHA} \\
\text { and } 0.27-0.45 \mathrm{~g} \mathrm{EPA} / \\
\text { d } \\
\text { Control group ( } \boldsymbol{n} \\
\text { 35): Not informed }\end{array}$ & $\begin{array}{l}\text { No adherence } \\
\text { protocol was } \\
\text { informed }\end{array}$ & $\begin{array}{l}\text { Intervention } \\
\text { group: } \\
45 \cdot 9 \pm 9 \cdot 3 \\
\text { Control } \\
\text { group: } \\
47 \cdot 3 \pm 12\end{array}$ & $\begin{array}{l}\text { Intervention } \\
\quad \text { group: } \\
34 \cdot 4 \pm 2 \cdot 69 \\
\text { Control } \\
\text { group: } \\
34 \cdot 7 \pm 3\end{array}$ & $\begin{array}{l}\text { LXA4 } \\
\text { LXA5 }\end{array}$ & $\begin{array}{l}n-6 \\
n-3\end{array}$ & $\begin{array}{l}50 \cdot 29 \pm 19 \cdot 10 \\
62 \cdot 3 \pm 24 \cdot 38\end{array}$ & $\begin{array}{l}57 \cdot 63 \pm 17 \cdot 83 \\
79 \cdot 8 \pm 31 \cdot 16\end{array}$ & $\begin{array}{l}P=0.069 \\
P=0.058\end{array}$ \\
\hline $\begin{array}{l}\text { Ramel, } 2010- \\
\text { Iceland, } \\
\text { Spain and } \\
\text { Ireland }^{(44)}\end{array}$ & $\begin{array}{l}\text { Randomised, } \\
\text { controlled dietary } \\
\text { intervention trial }\end{array}$ & 8 weeks & $\begin{array}{l}\text { Salmon and } \\
\text { oral oil } \\
\text { supplement }\end{array}$ & $\begin{array}{l}\text { Food intervention } \\
\text { group ( } \boldsymbol{n} 84): 150 \mathrm{~g} \\
\text { salmon, 3 times per } \\
\text { week. } 2 \cdot 1 \mathrm{~g} \text { LC } n-3 \\
\text { PUFA } \\
\text { Fish oil intervention } \\
\text { group }(\boldsymbol{n} \text { 80): } 1 \cdot 3 \mathrm{~g} \\
\text { EPA plus DHA } \\
\text { (6 cápsules per day) } \\
\text { Control group ( } \boldsymbol{n} \\
\text { 80): no seafood } \\
\text { (6 sunflower oil } \\
\text { capsules per day) }\end{array}$ & $\begin{array}{l}\text { Food Frequency } \\
\text { Questionnaire }\end{array}$ & 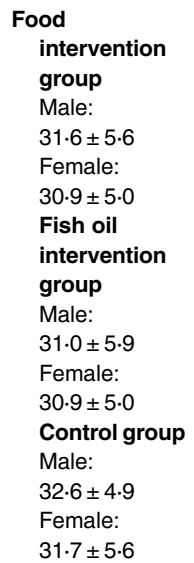 & 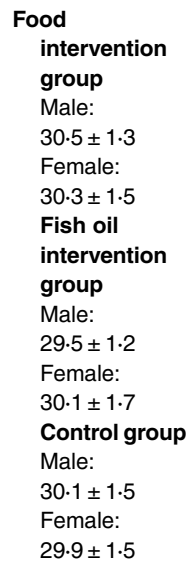 & PGF2 & $n-6$ & $\begin{array}{l}\text { Salmon } \\
\quad \text { Male: } \\
188 \pm 182 \\
\text { Female: } \\
202 \pm 199 \\
\text { Fish oil } \\
\text { Male: } \\
270 \pm 451 \\
\text { Female: } \\
228 \pm 321\end{array}$ & $\begin{array}{l}\text { Salmon } \\
\text { Male: } 170 \pm 197 \\
\text { Female: } \\
148 \pm 184 \\
\text { Fish oil } \\
\text { Male: } 189 \pm 141 \\
\text { Female: } \\
139 \pm 199\end{array}$ & $\begin{array}{l}P<0.05^{f} \\
P<0.05^{f}\end{array}$ \\
\hline
\end{tabular}

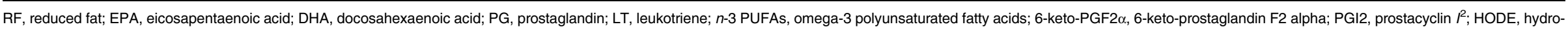

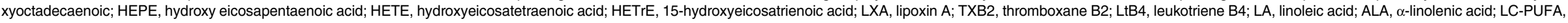
long-chain polyunsaturated fatty acid; LtB5, leukotriene B5; LOX, lipooxygenase enzymes; COX, cyclooxygenase enzymes; CYP450, cytochrome P450.

${ }^{a}$ All results are presented in $\mathrm{pg} / \mathrm{ml}$, except for Nielson et al. ( $\mathrm{ng} / 10^{7} \mathrm{cells}$ ) and O'Sullivan et al. with association analysis.

${ }^{\mathrm{b}}$ Compared with the control group.

${ }^{\circ}$ Compared within $n-3$ intervention groups.

d 5-HEPE (nM) v. erythrocytes EPA (mol\%) Linear regression analyses.

LTB4 (nM) v. erythrocytes EPA (mol\%) Linear regression analyses.

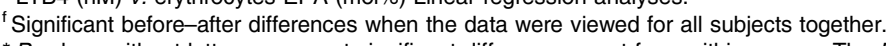

* $P$-values without letters represent significant difference or not from within groups. The $P$-value was extracted from the data of articles included in this review.

The bold values in (Significance column) represent the $p$ values that presented a significant result $(p<0.05)$. 


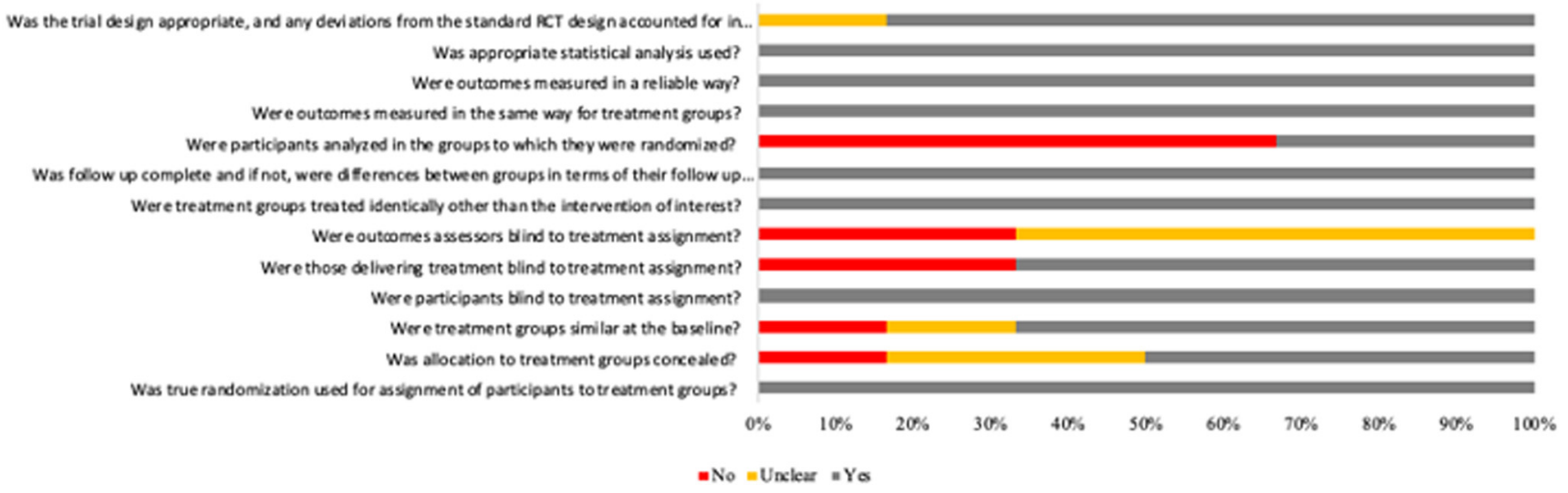

Fig. 2. Review of judgements of authors about each risk of bias item according to The Joanna Briggs Institute Critical Appraisal Checklist for Randomised Controlled Trials is presented as percentages across all included studies.

with olive oil ${ }^{(41)}$, maize/soyabean oil ${ }^{(42)}$ or sunflower oil capsules $^{(44)}$ and one study did not specify which oil was used $^{(43)}$. Studies that utilised food as PUFAs source presented conventional fruit yoghurt ${ }^{(39)}$ and normal-fat frankfurters and pates as control groups ${ }^{(38)}$.

The lower $n$-3 PUFA dose observed was $0 \cdot 25 \mathrm{~g}$ for $\mathrm{DHA}^{(40)}$ and the higher dose was $3 \mathrm{~g}$ for a combination of ALA, EPA, $\mathrm{DPA}$ and $\mathrm{DHA}^{(39,42)}$.

Each study measured a particular subset of eicosanoids, varying from leukotrienes, PG, thromboxanes, lipoxins to PUFA metabolites such as HEPEs and HETEs. Five of seven studies measured both pro- and anti-inflammatory eicosanoids ${ }^{(39-43)}$, and two studies measured only the pro-inflammatory ones ${ }^{(38,44)}$.

\section{Adherence protocols and analysis of cellular fatty acid content}

Different protocols were used in each of the studies in order to verify adherence to the intervention. Food frequency questionnaires ${ }^{(39,41,42,44)}$ and Dietary Register ${ }^{(38)}$ were used. Two studies did not mention the specific adherence protocol used ${ }^{(40,43)}$.

Five of seven studies analysed the fatty acid content after the intervention period, four of them used erythrocytes membrane ${ }^{(39,40,42,43)}$, one used neutrophils membrane ${ }^{(41)}$, and all of them used gas chromatography (GC) as the standard methodology to identify the fatty acid profile. All studies showed that n-3 PUFA intake led to a significant membrane fatty acid incorporation, suggesting compliance with the $n-3$ PUFA intervention protocol.

\section{Risk of bias within individual studies}

The Joanna Briggs Institute appraisal tools were used to assess risk of bias for all studies included. Of the six RCTs, two were judged to be at 'low risk of bias ${ }^{(39,41)}$. The non-randomised clinical trial ${ }^{(38)}$ and four $\mathrm{RCTs}^{(40,42-44)}$ were classified as being at 'high risk of bias'.

The included studies reported low risks of bias regarding randomisation, blindness, outcomes measured. Considering the randomised trials, $100 \%$ indicated a low risk of bias for randomisation of participants to the treatment group, blinding of participants to treatment assignment, identical groups other than the intervention of interest, complete follow-up, outcome measured in an equal and reliable way for treatment groups and appropriate statistical analysis. In all RCTs assessed, the one domain judged with a total high risk for bias was regarding intention-to-treat analysis, resulting in one 'unclear' ${ }^{(41)}$ and six 'no' answers ${ }^{(39,40,42-44)}$. In two RCTs, individuals delivering the treatment and outcome assessors were not blind to treatment assignment ${ }^{(40,44)}$. In only one RCT, the allocation of treatment groups was not concealed ${ }^{(44)}$. In all trials assessed, there was at least one domain judged with unclear risk for bias. Fig. 2 shows the individual risk of bias assessments for all studies included in this work.

\section{Results of individual studies}

Five of seven studies presented an overall reduction in pro-inflammatory eicosanoids after $n$-3 PUFA intervention, and a less pronounced effect in anti-inflammatory eicosanoids (Table 1). Only DeLuis et al. ${ }^{(34)}$ showed enhanced serum levels in AA-derived eicosanoids levels after $n$-3 PUFA intake.

COX-derived eicosanoids such as 6-keto-prostaglandin F1 $\alpha$ $(6 \text {-keto-PGF1 } \alpha)^{(38)}$, PGE2 $2^{(39,40)}$, prostaglandin D2 (PGD2) ${ }^{(39)}$, prostaglandin F2 (PGF2) ${ }^{(44)}$ and TXB2 ${ }^{(38)}$ presented lower serum levels after $n$-3 PUFA intervention. DeLuis et al. ${ }^{(40)}$ was the only study that presented opposite effects with an increase in PGE2 and TXB2 levels after EPA plus DHA supplementation. The eicosanoid prostaglandin E3 (PGE3) presented reduced serum levels after $n-3$ enriched yoghurt ${ }^{(39)}$.

Concerning the LOX-5-derived eicosanoids, lower serum levels of LTB4 were observed after $n-3$ PUFA supplementation in one study ${ }^{(41)}$, but interestingly DeLuis et al. ${ }^{(40)}$ observed higher levels after the intervention period. The HETE family such as 5-HETE, 8-HETE, 9-HETE, 11-HETE ${ }^{(39)}$ and 12-HETE ${ }^{(40)}$ showed reduced serum levels after $n-3$ PUFA intake. However, 15-HETE and 8-HETE presented increased serum levels after $n$-3 PUFA intervention in the study conducted by DeLuis et al. ${ }^{(40)}$. In only one study, 5-HEPE EPA-derived eicosanoid presented higher serum levels after intervention ${ }^{(41)}$.

\section{Synthesis of results}

Due to the available data, we were able to conduct a subgroup analysis with the arachidonic acid COX-derived PG eicosanoid 


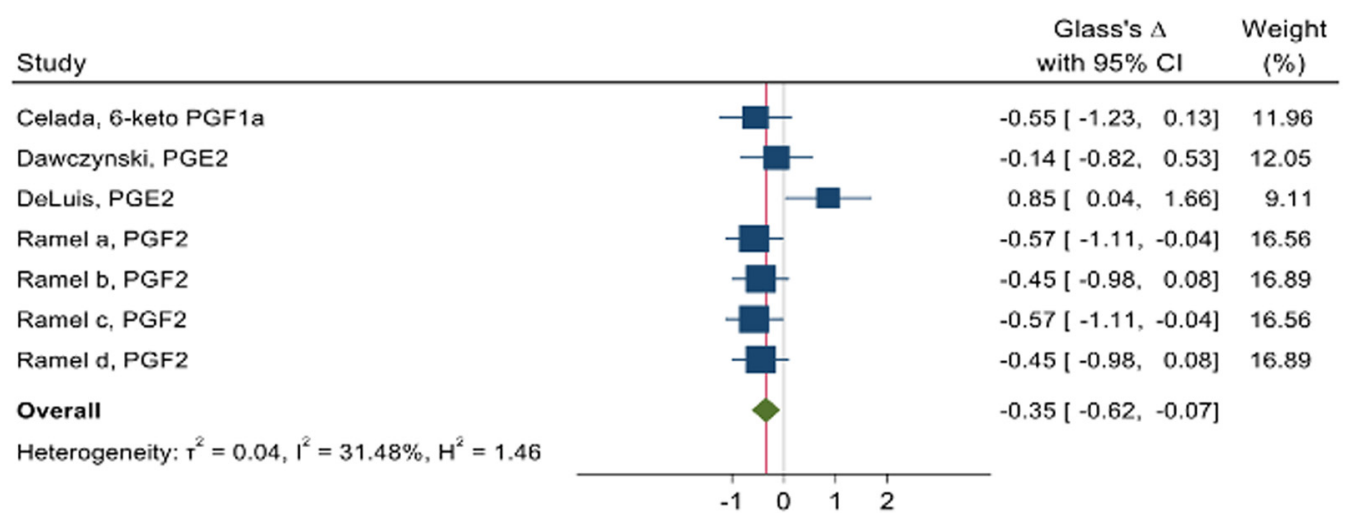

Fig. 3. Pooled effect size of $n$-3 PUFA intake on COX-derived prostaglandins markers in individuals with obesity and overweight. Pooled effect estimates from meta-analysis are expressed as standardised mean differences (SMD), represented by diamonds. The $95 \% \mathrm{Cls}$ is the line through the diamond and were estimated with the use of a generic inverse variance random-effect model. Interstudy heterogeneity was detected with the use of Cochran's $Q$ statistic and quantified with the use of the $I^{2}$ statistic. 6-keto-PGF1 $\alpha$, 6-keto-prostaglandin F1alpha; PGE2, prostaglandin E2; PGF2, prostaglandin F2. $n$-3 sources: Celada: $n$-3 enriched paté group; Dawczynski: $n$-3 enriched yoghurt group; DeLuis: DHA supplementation group; Ramel a: fish oil male group; Ramel b: fish oil female group; Ramel c: salmon male group; Ramel d: salmon female group.

Table 2. Subgroup analysis for the effect of and $n$-3 PUFA intake on COX-derived prostaglandins profile on subjects with obesity and overweight

\begin{tabular}{lcccrr}
\hline Subgroups & $N$ & Glass's $\Delta$ & $95 \% \mathrm{Cl}$ & $P^{2}(\%)$ & $P\left(\chi^{2}\right)$ \\
\hline $\begin{array}{l}\text { Overall } \\
n \text {-3 PUFA dose }\end{array}$ & 7 & -0.35 & $-0.62,-0.07$ & 31.48 & - \\
$\quad$ High & 5 & $-\mathbf{0 . 4 6}$ & $-\mathbf{0 . 7 2 , - 0 . 2 7}$ & 0.00 & 0.88 \\
$\quad$ Low & 2 & 0.13 & $-1.24,1.50$ & 85.16 & 0.01 \\
$\quad \begin{array}{l}\text { Time of intervention } \\
\quad \text { More than 8 weeks }\end{array}$ & 2 & 0.33 & $-0.65,1.30$ & 70.74 & 0.06 \\
$\quad \begin{array}{l}\text { Up to 8 weeks } \\
n-3 \text { PUFA source }\end{array}$ & 5 & $-\mathbf{0 . 5 1}$ & $-\mathbf{0 . 7 6 , - 0 . 2 7}$ & 0.00 & 0.99 \\
$\quad$ Food & & -0.34 & $-0.83,0.13$ & 0.00 & 0.41 \\
$\quad$ Oil Supplement & 5 & -0.31 & $-0.73,0.12$ & 63.17 & 0.04 \\
\hline
\end{tabular}

PUFA, polyunsaturated fatty acid; $\mathrm{Cl}$, confidence interval.

The bold values in (Significance column) represent the $p$ values that presented a significant result $(p<0.05)$

group. Meta-analysis presented an overall reduction in PG series (Glass's $\Delta-0 \cdot 35 ; 95 \% \mathrm{CI}-0 \cdot 62,-0 \cdot 07$ ) after $n-3$ PUFA intake (Fig. 3).

\section{Subgroup analysis}

Subgroup analysis showed significant effects by reducing arachidonic acid COX-derived PG levels when $n$-3 PUFA was consumed in higher doses (Glass's $\Delta-0 \cdot 46 ; 95 \%$ CI $-0.71,-0.21)$ and with the period of intervention up to 8 weeks (Glass's $\Delta-0 \cdot 35 ; 95 \%$ CI $-0 \cdot 62,-0 \cdot 07$ ). There was no difference in arachidonic acid COX-derived PG levels when food (Glass's $\Delta-0 \cdot 34 ; 95 \%$ CI $-0 \cdot 82,0 \cdot 13$ ) or oil supplement (Glass's $\Delta-0 \cdot 31 ; 95 \%$ CI $-0.73,0 \cdot 12$ ) was taken into consideration. More details are shown in Table 2.

\section{Discussion and conclusions}

The studies presented in this systematic review suggest the anti-inflammatory effects of $n-3$ PUFA intake on serum eicosanoid profile and, for the first time, in individuals with obesity and overweight. The meta-analysis result showed an overall reduction on arachidonic acid COX-derived PG serum levels. Also, a reduction was seen in doses higher than $0.5 \mathrm{~g} / \mathrm{d}$ of $\mathrm{EPA}+\mathrm{DHA}$ and with the period of intervention less than 8 weeks. This is an important finding considering that $n-3$ PUFA could improve metabolic alterations by mediating chronic low-grade inflammation related to obesity.

The daily supply of $n$-3 PUFAs either by supplementation or enriched food by diet are recommended by several agencies and organisations since previous studies suggest their protective effect on cardiovascular diseases ${ }^{(45,46)}$ and cancer ${ }^{(47,48)}$. The World Health Organization (WHO, 2000) recommends $1-2 \%$ of daily energy consumption from ômega-3 PUFA for the general population and the International Society for the Studies of Fatty Acids and Lipids ${ }^{(37)}$ recommend at least $500 \mathrm{mg} / \mathrm{d}$ of EPA + DHA for the general adult population aiming for cardiovascular health. However, there is no current daily recommendation for adults with obesity and overweight, but it is noticed that the excess of fat, especially the visceral fat, increases risk for cardiovascular diseases and other comorbidities ${ }^{(49,50)}$. Actually, the ratio of dietary $n-6$ to $n-3$ PUFA, rather than the absolute amount of $n-3$ PUFA, is important in determining the development of inflammatory eicosanoid response. Based on the understanding that $n-6$ PUFA induces a more potent inflammatory response, whereas $n-3$ PUFA are thought to have a less potent inflammatory effect, the fluctuation in $n-6 / n-3$ ratio intake contributes to the eicosanoid profile release from adipose tissues. When pro-inflammatory eicosanoids are enhanced, they can unbalance the inflammatory signal and promote the recruitment of M1-polarized macrophages, increasing pro-inflammatory adipokines and cytokines secretion by adipose tissue ${ }^{(46,47)}$. Given the inefficiency of transformation from dietary ALA to $n$-3 long-chain PUFA $^{(19)}$, increasing dietary consumption of EPA and DHA helps to reach the maximum beneficial effects, reducing the inflammatory response in individuals with obesity ${ }^{(51,52)}$.

Investigation about mechanisms underlying the attenuation of inflammatory response and metabolic dysfunction in individuals with obesity by $n-3$ PUFA intake are progressing over 
the last decades. Anti-inflammatory effects of $n$-3 PUFA are primarily demonstrated by two main mechanisms: inhibitory secretion of pro-inflammatory mediators and greatly reduced macrophage migration into adipose tissue. The effect in obese animal models (high-fat diet-induced obesity and genetic obesity) is promising, but the proposed mechanisms still require further confirmation in humans. Evidence from in vitro and in vivo studies suggests that EPA and DHA can reduce pro-inflammatory adipokine synthesis, decreasing the inflammatory crosstalk between adipocytes and infiltrative immune cells ${ }^{(26)}$, murine macrophages ${ }^{(53)}$ and mice CD8 lymphocytes $^{(54)}$. In adipocytes, the peroxisome proliferator-activated receptor gamma (PPAR $\gamma$ ) functions as an effector of $T$ helper 2 cytokines (Th2), whose activation is necessary for the differentiation of circulating monocytes into M2 macrophages and transcription of anti-inflammatory genes. PUFA $n$-3 substitution of AA in adipocyte membrane phospholipids results in a decrease in the level of PGE2 and a subsequent reduction in the enzymatic activity of fatty acid synthesis ${ }^{(55)}$, which restricts adipocyte hypertrophy. On the other hand, treatment with $n$-3 PUFA results in a significant decrease in the size of mature adipocytes and accumulation of smaller adipocytes in obese diabetic mice ${ }^{(56)}$, and the decrease in the formation of PGD2 and its derivatives, known as PPAR $\gamma$ ligands, explains the effects of $n-3$ PUFAs on the proliferation and maturation of adipocytes ${ }^{(57)}$. However, the exact mechanisms of the interaction of $n$-3 PUFA effects between immune cells and adipocytes need to be explored.

White adipocytes have an important role in the orchestrating inflammatory response in white adipose tissue, by releasing several pro-inflammatory molecules and activating and recruiting immune cells ${ }^{(58)}$. The COX-1 and COX-2 enzymes are involved in the inflammation regulatory process ${ }^{(53,54)}$. Thus, a pro-inflammatory microenvironment leads to a higher release of pro-inflammatory eicosanoids from AA, especially from the prostaglandin family. The breast tissue of women with obesity was demonstrated that a higher concentration of pro-inflammatory cytokines promotes greater macrophage COX-2 expression and produces more PGE $2^{(59)}$. The present study clearly links obesity and low-grade chronic inflammation, processes mediated by COX-2 and aromatase expression in human breast tissue.

Studies have demonstrated that $n$-3 PUFA may replace AA and shift the eicosanoid profile, but distinct results have been found depending on which type of $n-3$ fatty acid is being offered to the studied population. In vitro study showed that EPA and DHA replaced $25-50 \%$ of AA in phospholipids on macrophage cellular membrane, and the AA fatty acids replace significantly reduced $50-65 \%$ the PGE2, TXB2 and 6-keto-PGF1 $\alpha$ synthesis $^{(55)}$. The study conducted by Nielsen and colleagues with a duration of 6 weeks offered an oral supplementation of both EPA and DHA and found significant reductions in LTB4 serum levels when subjects in the intervention group was compared with their respective controls. These results were also seen in individuals with obesity with EPA $0.64 \mathrm{~g} / \mathrm{d}$ and DHA $0.48 \mathrm{~g} / \mathrm{d}$ supplementation ${ }^{(35)}$ and with EPA $2 \mathrm{~g} / \mathrm{d}$ and DHA $1 \mathrm{~g} / \mathrm{d}$ in overweight subjects ${ }^{(36)}$, which are considered low and high doses, respectively. Interestingly, the study conducted by De Luis et al. ${ }^{(34)}$ presented contrasting results regarding eicosanoids synthesis. It revealed an increase in LTB4, 8-HETE and 15-HETE after $0.5 \mathrm{~g} / \mathrm{d}$ DHA supplementation for 6 months concomitantly with a very low-calorie ketogenic diet. According to the authors, adipose tissue is considered as a source of NEFA and fatty acid-derived bioactive inflammatory lipid mediators and during the intervention period of significant weight loss, these may be released from adipose tissue to systemic circulation. Also, it needs to be considered that the protocol supplementation was composed solely by DHA (did not include EPA), which may influence the eicosanoid profile synthesis, since DHA is a major precursor of docosanoids, a similar but distinct subset of biomolecules involving in the inflammatory response.

Evaluating the PG group, meta-analysis presented a significant reduction in arachidonic acid COX-derived PG when intake was higher than $0.5 \mathrm{~g} / \mathrm{d} \mathrm{EPA}+\mathrm{DHA}$, regardless. This is in accordance with previous studies that stated a dosedependent immunological response between $n$-3 intake and PGE2 and PGE3 synthesis ${ }^{(21,60)}$. There is also evidence in humans, suggesting that an intake between 1.35 and $2.7 \mathrm{~g}$ $n$-3 PUFA would be required to affect PGE2 production by mononuclear cells in healthy younger and older $\operatorname{men}^{(60)}$. Despite the non-significant effect from the source of $n-3$ PUFA, provided by a food source or oil supplement, it is known the better bio-utilisation of lipids from foods can be attributed to the larger amount of fat as part of the natural composition of food, favouring lipid absorption and conferring a higher bioavailability ${ }^{(61)}$.

Time is a critical factor when it comes to $n-3$ PUFA effectiveness, since the benefits over inflammation appear to be dependent on the incorporation of fatty acids in the cell membrane ${ }^{(62)}$ and that process might take about 3-4 weeks to reach its peak ${ }^{(63)}$. It is important to mention that the studies included in meta-analysis started from a 4 -week period intervention, which comprehends sufficient time to incorporate $n-3$ fatty acids on the cell membrane. Unexpectedly, a significant reduction in arachidonic acid COX-derived PG was showed when the time of intervention was less than 8 weeks, a pattern that could not be seen for more prolonged periods (more than 8 weeks). That outcome can be associated with the different doses used in each protocol. It means that higher doses for shorter periods could elicit a significant effect on the eicosanoid profile, whereas lower doses for more prolonged periods could not. Studies measuring PG after $n-3$ PUFA supplementation in periods shorter than 8 weeks are scarce. Tecklenburg-Lund et al. ${ }^{(64)}$ showed that $3.2 \mathrm{~g}$ EPA plus $2.0 \mathrm{~g}$ DHA oral supplementation taken daily for 3 weeks was effective in reducing $11 \beta$-prostaglandin F2 in asthmatic individuals. However, a reasonable amount of evidence shows beneficial effects of $n-3$ PUFAs for more prolonged periods, with reductions in PG being observed even at the third ${ }^{(65,66)}$ and sixth month ${ }^{(67)}$ of intervention.

One of the challenges to understand the effects of $n-3$ PUFA in the inflammatory response in obesity is related to 
the wide variety of $n-3$ PUFA intervention protocols used in human trials. Those include dose, source of $n$-3 PUFA, distribution and amounts of total and subtypes fatty acids in the $n-3$ PUFA source, population evaluated, genetic backgrounds, environmental conditions, quality and quantity of the diet, etc $^{(68)}$. Besides that, the baseline values of anti-inflammatory eicosanoids may mask the real effect of PUFA supplementation. Also, individual responsiveness to $n-3$ fatty acids should be taken into account as another major limitation to assess the effect on the inflammatory process and to suggest an optimal dose for $n$-3 PUFA.

The present study has strengths, including (i) an effort was made to search for data in seven different databases and rigorously following PRISMA directions in order to minimise publication bias; (2) utilisation of validated tools to characterise included studies in terms of methodological quality; and (3) the summarised pool analysis focused on studies measuring comparable outcomes with similar protocols, reducing methodological heterogeneity. Additionally, there are limitations in the present study. Firstly, our meta-analysis results used the delta values within the same group, and not between control and intervention groups. Secondly, the different types of $n-3$ fatty acids could be observed in intervention groups, and that may influence the final results, since there is a discrepant rate of interconversion between ALA, EPA, DPA and DHA fatty acids ${ }^{(69)}$. Lastly, due to the limited trials, the present study included a small number of clinical trials $(n 7)$, and therefore, more well-conducted controlled trials are required to strengthen our findings and explore optimal doses and period intake in individuals with overweight and obesity.

In conclusion, this systematic review and meta-analysis evidence the effect of $n-3$ PUFA intake through diet or supplementation on eicosanoid synthesis, suggesting a benefit reduction on arachidonic acid COX-derived PG in individuals with obesity and overweight. Finally, $n-3$ PUFA intake may be an interesting strategy to be considered by healthcare professionals in association with a multifaceted approach to manage obesity such as a healthy eating pattern, achieving and maintaining a healthy weight and getting regular physical activity, in order to soften low-grade chronic inflammatory response in obesity treatment.

\section{Acknowledgements}

The authors acknowledge Eliane Said Dutra for her contribution to the PRESS protocol.

G. R. B. S., I. N. M. S. R., V. S. S. G. and N. P. contributed significantly to the work's conception, design, data collection, interpretation and analysis; G. R. B. S., I. N. M. S. R., V. S. S. G., K. G. M. and N. P. participated in the writing and critical revision of the article in a manner sufficient to establish ownership of the intellectual content; and G. R. B. S., I. N. M. S. R., V. S. S. G., K. G. M. and N. P. read and approved the final version of the manuscript.

This research did not receive any specific grant from funding agencies in the public, commercial or not-for-profit sectors.

The authors declare that they have no conflicts of interest.

\section{References}

1. WHO Consultation on Obesity (1999: Geneva, Switzerland) \& World Health Organization. (2000) Obesity: preventing and managing the global epidemic: report of a WHO consultation. World Health Organization. https://apps.who.int/iris/handle/10665/ 42330.

2. Pickens CA, Sordillo LM, Zhang C, et al. (2017) Obesity is positively associated with arachidonic acid-derived 5- and 11-hydroxyeicosatetraenoic acid (HETE). Metabolism 70, 177-191.

3. Geo B, Lang S, Duan Y, et al. (2019) Serum and fecal oxylipins in patients with alcohol-related liver disease. Dig Dis Sci 64, 18781892. doi:10.1007/s10620-019-05638-y.

4. Djuric Z, Turgeon DK, Sen A, et al. (2017) The anti-inflammatory effect of personalized omega- 3 fatty acid dosing for reducing prostaglandin $\mathrm{E}_{2}$ in the colonic mucosa is attenuated in obesity. Cancer Prev Res 10, 729-737. doi:10.1158/1940-6207.CAPR-17-0091.

5. Hotamisligil GS (2006) Inflammation and metabolic disorders. Nature 444, 860-867. doi:10.1038/nature05485.

6. Ying W, Wollam J, Ofrecio JM, et al. (2017) Adipose tissue B2 cells promote insulin resistance through leukotriene LTB4/LTB4R1 signaling. J Clin Invest 127, 1019-1030. doi:10.1172/JCI90350.

7. Wang D \& DuBois RN (2012) Associations between obesity and cancer: the role of fatty acid synthase. JNCI J Natl Cancer Inst 104, 343-345. doi:10.1093/jnci/djs010.

8. Nasrallah R, Hassouneh R \& Hébert RL (2016) PGE2, kidney disease, and cardiovascular risk: beyond hypertension and diabetes. $J$ Am Soc Nephrol 27, 666-676. doi:10.1681/ASN.2015050528.

9. Vianello E, Dozio E, Bandera F, et al. (2020) Correlative study on impaired prostaglandin E2 regulation in epicardial adipose tissue and its role in maladaptive cardiac remodeling via EPAC2 and ST2 signaling in overweight cardiovascular disease subjects. Int J Mol Sci 21. doi:10.3390/ijms21020520.

10. Ferrante AW (2007) Obesity-induced inflammation: a metabolic dialogue in the language of inflammation. J Intern Med 262, 408414. doi:10.1111/j.1365-2796.2007.01852.x.

11. Titos E, Rius B, Lopez-Vicario C, et al. (2016) Signaling and immunoresolving actions of resolvin D1 in inflamed human visceral adipose tissue. J Immunol 197, 3360-3370. doi:10.4049/jimmunol.1502522.

12. Nettleton JA \& Katz R (2005) $n-3$ long-chain polyunsaturated fatty acids in type 2 diabetes: a review. $J$ Am Diet Assoc 105, 428-440. doi:10.1016/j.jada.2004.11.029.

13. Simopoulos AP (2006) Evolutionary aspects of diet, the omega-6/ omega-3 ratio and genetic variation: nutritional implications for chronic diseases. Biomed Pharmacother 60, 502-507. doi:10.1016/j. biopha.2006.07.080.

14. Mas E, Croft KD, Zahra P, et al. (2012) Resolvins D1, D2, and other mediators of self-limited resolution of inflammation in human blood following $n-3$ fatty acid supplementation. Clin Chem 58, 1476-1484. doi:10.1373/clinchem.2012.190199.

15. Gropper SS, Smith JL \& Groff JL (2013) Advanced Nutrition and Human Metabolism, 6th ed, Boston, MA: Cengage Learning.

16. Bowers LW \& DeGraffenried LA (2015) Targeting the COX-2 pathway to improve therapeutic response in the obese breast cancer patient population. Curr Pharmacol Rep 1, 336-345. doi:10.1007/ s40495-015-0041-y.

17. Wang Y, Yan S, Xiao B, et al. (2018) Prostaglandin F $2 \alpha$ facilitates hepatic glucose production through CaMKII $\gamma / \mathrm{p} 38 / \mathrm{FOXO} 1$ signaling pathway in fasting and obesity. Diabetes 67, 1748-1760. doi:10.2337/db17-1521.

18. Micallef M, Munro I, Phang M, et al. (2009) Plasma $n$-3 polyunsaturated fatty acids are negatively associated with obesity. $\mathrm{Br} J$ Nutr 102, 1370-1374. doi:10.1017/S0007114509382173.

19. Arterburn LM, Hall EB \& Oken H (2006) Distribution, interconversion, and dose response of $n-3$ fatty acids in humans. $A m \mathrm{~J}$ Clin Nutr 83. doi:10.1093/ajcn/83.6.1467s.

20. Leung PS \& Zhang D (2014) Potential roles of GPR120 and its agonists in the management of diabetes. Drug Des Dev Ther 1013. doi:10.2147/DDDT.S53892. 
21. Fischer R, Konkel A, Mehling H, et al. (2014) Dietary omega-3 fatty acids modulate the eicosanoid profile in man primarily via the CYP-epoxygenase pathway. J Lipid Res 55, 1150-1164. doi:10.1194/ jlr.M047357.

22. Calder PC (2015) Marine omega-3 fatty acids and inflammatory processes: effects, mechanisms and clinical relevance. $B B A-M o l$ Cell Biol Lipids 1851, 469-484. doi:10.1016/j.bbalip.2014.08.010.

23. Wang W, Yang J, Yang H, et al. (2016) Effects of high-fat diet on plasma profiles of eicosanoid metabolites in mice. Prostaglandins Other Lipid Mediat 127, 9-13. doi:10.1016/j.prostaglandins.2016.11.003.

24. Onodera T, Fukuhara A, Shin J, et al. (2017) Eicosapentaenoic acid and 5-HEPE enhance macrophage-mediated Treg induction in mice. Sci Rep 7, 4560. doi:10.1038/s41598-017-04474-2.

25. Browning LM (2003) $n-3$ polyunsaturated fatty acids, inflammation and obesity-related disease. Proc Nutr Soc 62, 447-453. doi:10.1079/ pns2003252.

26. Calder PC (2003) n-3 polyunsaturated fatty acids and inflammation: from molecular biology to the clinic. Lipids 38, 343-352. doi:10.1007/s11745-003-1068-y.

27. Jiang J, Li K, Wang F, et al. (2016) Effect of marine-derived n-3 polyunsaturated fatty acids on major eicosanoids: a systematic review and meta-analysis from 18 randomized controlled trials. PLOS ONE 11, 1-18. doi:10.1371/journal.pone.0147351.

28. Moher D, Shamseer L, Clarke M, et al. (2015) Preferred reporting items for systematic review and meta-analysis protocols (PRISMA-P) 2015 statement. Syst Rev 4, 1. doi:10.1186/2046-4053-4-1.

29. Ouzzani M (2017) Rayyan - a web and mobile app for systematic reviews. Syst Rev 2016, 1-10. doi:10.1186/s13643-016-0384-4.

30. Raudenbush SW (2009) Analyzing effect sizes: random-effects models. In The Handbook of Research Synthesis and Meta-analysis, pp. 295-315 [H Cooper, LV Hedges \& JC Valentine Eds.]. New York: Russell Sage Foundation.

31. DerSimonian R \& Laird N (1986) Meta-analysis in clinical trials. Control Clin Trials 7, 177-188. doi:10.1016/0197-2456(86)90046-2.

32. Deeks JJ, Higgins JP \& Altman DG (2019) Analysing data and undertaking meta-analyses. In Cochrane Handbook for Systematic Reviens of Interventions, pp. 241-284. Wiley. doi:10.1002/ 9781119536604.ch10

33. Smith ML\& Glass GV (1977) Meta-analysis of psychotherapy outcome studies. Am Psychol 32, 752-760. doi:10.1037/0003-066X.32.9.752.

34. Kline RB (2013) Beyond Significance Testing: Statistics Reform in the Behavioral Sciences, 2nd ed., Washington, DC: American Psychological Association.

35. Higgins JPT\& Green S (2011) Cocbrane Handbook for Systematic Reviens of Interventions. 5.1.0. The Cochrane Collaboration.

36. Higgins JPT, Thomas J, Chandler J, et al. (2020) Cochrane Handbook for Systematic Reviews of Interventions. 6.1. Cochrane.

37. ISSFAL (2004) Recommendations for Intake of Polyunsaturated Fatty Acids in Healtby Adults.

38. Celada P, Olmedilla-Alonso B, Delgado-Pando G, et al. (2019) Coagulation, thrombogenesis, and insulin resistance markers in increased-cardiovascular-risk subjects consuming improved-fat meat products. J Am Coll Nutr 38, 334-341. doi:10.1080/ 07315724.2018.1513345.

39. Dawczynski C, Massey KA, Ness C, et al. (2013) Randomized placebo-controlled intervention with $n$-3 LC-PUFA-supplemented yoghurt: effects on circulating eicosanoids and cardiovascular risk factors. Clin Nutr 32, 686-696. doi:10.1016/j.clnu.2012.12.010.

40. de Luis D, Domingo JC, Izaola O, et al. (2016) Effect of DHA supplementation in a very low-calorie ketogenic diet in the treatment of obesity: a randomized clinical trial. Endocrine 54, 111-122. doi:10.1007/s12020-016-0964-z.

41. Nielsen MS, Gammelmark A, Madsen T, et al. (2012) The effect of low-dose marine $n$-3 fatty acids on the biosynthesis of pro-inflammatory 5-lipoxygenase pathway metabolites in overweight subjects: a randomized controlled trial. Prostaglandins Leukot Essent Fatty Acids 87, 43-48. doi:10.1016/j.plefa.2012.05.009.

42. O'Sullivan A, Armstrong P, Schuster GU, et al. (2014) Habitual diets rich in dark-green vegetables are associated with an increased response to $\omega-3$ fatty acid supplementation in Americans of African Ancestry. J Nutr 144, 123-131. doi:10.3945/jn.113.181875.

43. Polus A, Zapala B, Razny U, et al. (2016) Omega-3 fatty acid supplementation influences the whole blood transcriptome in women with obesity, associated with pro-resolving lipid mediator production. Biochim Biophys Acta 1861, 1746-1755. doi:10.1016/j. bbalip.2016.08.005

44. Ramel A, Martinez JA, Kiely M, et al. (2010) Effects of weight loss and seafood consumption on inflammation parameters in young, overweight and obese European men and women during 8 weeks of energy restriction. Eur J Clin Nutr 64, 987-993. doi:10.1038/ ejcn.2010.99.

45. Harris WS, Poston WC \& Haddock CK (2007) Tissue $n-3$ and $n-6$ fatty acids and risk for coronary heart disease events. Atherosclerosis 193, 1-10. doi:10.1016/j.atherosclerosis.2007.03.018.

46. Kark JD, Kaufmann NA, Binka F, et al. (2003) Adipose tissue $n-6$ fatty acids and acute myocardial infarction in a population consuming a diet high in polyunsaturated fatty acids. Am J Clin Nutr 77, 796-802. doi:10.1093/ajen/77.4.796.

47. Zock PL \& Katan MB (1998) Linoleic acid intake and cancer risk: a review and meta-analysis. Am J Clin Nutr 68, 142-153. doi:10.1093/ajen/68.1.142.

48. Goodstine SL, Zheng T, Holford TR, et al. (2003) Dietary (n-3)/ (n-6) fatty acid ratio: possible relationship to premenopausal but not postmenopausal breast cancer risk in U.S. women. $J$ Nutr 133, 1409-1414. doi:10.1093/jn/133.5.1409.

49. Van Gaal LF, Mertens IL \& De Block CE (2006) Mechanisms linking obesity with cardiovascular disease. Nature 444, 875-880. doi:10.1038/nature05487.

50. Simopoulos AP (2016) An increase in the omega-6/omega-3 fatty acid ratio increases the risk for obesity. Nutrients 8, 128. doi: $10.3390 /$ nu8030128.

51. Ricciotti E \& Fitzgerald GA (2012) Prostaglandins and Inflammation. Arter Thromb Vasc Biol 31, 986-1000. doi:10.1161/ ATVBAHA.110.207449.

52. Libby P (2007) Inflammatory mechanisms: the molecular basis of inflammation and disease. Nutr Rev 2007. doi:10.1301/nr.2007. dec.S140

53. De Boer AA, Monk JM \& Robinson LE (2014) Docosahexaenoic acid decreases pro-inflammatory mediators in an in vitro murine adipocyte macrophage co-culture model. PLoS ONE 9, e85037. doi:10.1371/journal.pone.0085037.

54. Liddle DM, Monk JM, Hutchinson AL, et al. (2019) CD8+ t cell/ adipocyte inflammatory cross-talk and ensuing M1 macrophage polarization are reduced by fish oil-derived $n-3$ polyunsaturated fatty acids, in part by a TNF- $\alpha$-dependent mechanism. J Nutr Biochem 108243. doi:10.1016/j.jnutbio.2019.108243.

55. Wortman P, Miyazaki Y, Kalupahana NS, et al. (2009) $n 3$ and $n 6$ polyunsaturated fatty acids differentially modulate prostaglandin $\mathrm{E}$ secretion but not markers of lipogenesis in adipocytes. Nutr Metab 6, 1-10. doi:10.1186/1743-7075-6-5.

56. Huber J, Löffler M, Bilban M, et al. (2007) Prevention of high-fat diet-induced adipose tissue remodeling in obese diabetic mice by n-3 polyunsaturated fatty acids. Int J Obes 31, 1004-1013. doi:10.1038/sj.ijo.0803511.

57. Flachs P, Rossmeisl M, Bryhn M, et al. (2009) Cellular and molecular effects of $n-3$ polyunsaturated fatty acids on adipose tissue biology and metabolism. Clin Sci (Lond) 116, 1-16. doi:10.1042/ CS20070456.

58. Liddle DM, Hutchinson AL, Wellings HR, et al. (2017) Integrated immunomodulatory mechanisms through which long-chain $n-3$ polyunsaturated fatty acids attenuate obese adipose tissue dysfunction. Nutrients 9. doi:10.3390/nu9121289.

59. Subbaramaiah K, Morris PG, Zhou XK, et al. (2013) Increased levels of COX-2 and prostaglandin E2 contribute to elevated aromatase expression in inflamed breast tissue of obese women. Cancer Discov 2, 356-365. doi:10.1158/2159-8290.CD-11-0241.

60. Rees D, Miles EA, Banerjee T, et al. (2006) Dose-related effects of eicosapentaenoic acid on innate immune function in healthy 
humans: a comparison of young and older men. Am J Clin Nutr 83, 331-342. doi:10.1093/ajcn/83.2.331.

61. Visioli F, Risé P, Barassi MC, et al. (2003) Dietary intake of fish vs. formulations leads to higher plasma concentrations of $n$-3 fatty acids. Lipids 38, 415-418. doi:10.1007/s11745-003-1077-x.

62. Mocellin MC, Camargo CQ, Araujo E, et al. (2015) A systematic review and meta-analysis of the $n-3$ polyunsaturated fatty acids effects on inflammatory markers in colorectal cancer. Clin Nutr. doi:10.1016/j.clnu.2015.04.013.

63. Cockbain AJ, Toogood GJ \& Hull MA (2012) Omega-3 polyunsaturated fatty acids for the treatment and prevention of colorectal cancer. Recent Adv Basic Sci. doi:10.1136/gut.2010.233718.

64. Tecklenburg-lund S, Mickleborough TD, Turner LA, et al. (2010) Randomized controlled trial of fish oil and montelukast and their combination on airway inflammation and hyperpnea-induced bronchoconstriction. PLOS ONE 5. doi:10.1371/journal.pone. 0013487.
65. Fortin PR, Lew RA, Liang MH, et al. (1995) Validation of a meta-analysis: the effects of fish oil in rheumatoid arthritis. $J$ Clin Epidemiol 48, 1379-1390. doi:10.1016/0895-4356(95)00028-3.

66. Goldberg RJ \& Katz J (2007) A meta-analysis of the analgesic effects of omega-3 polyunsaturated fatty acid supplementation for inflammatory joint pain. Pain 129, 210-223. doi:10.1016/j.pain.2007.01.020.

67. Vedin I, Cederholm T, Freund-levi Y, et al. (2010) Reduced prostaglandin $\mathrm{F}_{2 \alpha}$ release from blood mononuclear leukocytes after oral supplementation of $\omega 3$ fatty acids: the OmegAD study. J Lipid Res 51, 1179-1185. doi:10.1194/jlr.M002667

68. De Mello AH, Uberti MF, De Farias BX, et al. (2018) N-3 PUFA and obesity: from peripheral tissues to the central nervous system. Br J Nutr 119, 1312-1323. doi:10.1017/S0007114518000429.

69. Innes JK \& Calder PC (2018) The differential effects of eicosapentaenoic acid and docosahexaenoic acid on cardiometabolic risk factors: a systematic review. Int J Mol Sci 9, 532. doi:10.3390/ ijms19020532. 


\begin{tabular}{|c|c|c|c|}
\hline Section/topic & \# & Checklist item & Reported on page \# \\
\hline \multicolumn{4}{|l|}{ TITLE } \\
\hline Title & 1 & Identify the report as a systematic review, meta-analysis, or both. & 1 \\
\hline \multicolumn{4}{|l|}{ ABSTRACT } \\
\hline Structured summary & 2 & $\begin{array}{l}\text { Provide a structured summary including, as applicable: background; objectives; data } \\
\text { sources; study eligibility criteria, participants, and interventions; study appraisal and } \\
\text { synthesis methods; results; limitations; conclusions and implications of key findings; } \\
\text { systematic review registration number. }\end{array}$ & $1-2$ \\
\hline \multicolumn{4}{|l|}{ INTRODUCTION } \\
\hline Rationale & 3 & Describe the rationale for the review in the context of what is already known. & $2-3$ \\
\hline Objectives & 4 & $\begin{array}{l}\text { Provide an explicit statement of questions being addressed with reference to } \\
\text { participants, interventions, comparisons, outcomes, and study design (PICOS). }\end{array}$ & 3 \\
\hline \multicolumn{4}{|c|}{ r } \\
\hline $\begin{array}{l}\text { Protocol and } \\
\text { registration }\end{array}$ & 5 & $\begin{array}{l}\text { Indicate if a review protocol exists, if and where it can be accessed (e.g. Web address), } \\
\text { and, if available, provide registration information including registration number. }\end{array}$ & 4 \\
\hline Eligibility criteria & 6 & $\begin{array}{l}\text { Specify study characteristics (e.g. PICOS, length of follow-up) and report characteristics } \\
\text { (e.g. years considered, language, publication status) used as criteria for eligibility, } \\
\text { giving rationale. }\end{array}$ & $4-5$ \\
\hline Information sources & 7 & $\begin{array}{l}\text { Describe all information sources (e.g. databases with dates of coverage, contact with } \\
\text { study authors to identify additional studies) in the search and date last searched. }\end{array}$ & $4-6$ \\
\hline Search & 8 & $\begin{array}{l}\text { Present full electronic search strategy for at least one database, including any limits } \\
\text { used, such that it could be repeated. }\end{array}$ & $\begin{array}{l}\text { 4, Figure } 1 \text { and Supplementary } \\
\text { Appendix S1 }\end{array}$ \\
\hline Study selection & 9 & $\begin{array}{l}\text { State the process for selecting studies (i.e. screening, eligibility, included in systematic } \\
\text { review, and, if applicable, included in the meta-analysis). }\end{array}$ & $\begin{array}{l}\text { 4-6, Supplementary } \\
\text { Appendix S1 }\end{array}$ \\
\hline $\begin{array}{l}\text { Data collection } \\
\text { process }\end{array}$ & 10 & $\begin{array}{l}\text { Describe method of data extraction from reports (e.g. piloted forms, independently, in } \\
\text { duplicate) and any processes for obtaining and confirming data from investigators. }\end{array}$ & 5 \\
\hline Data items & 11 & $\begin{array}{l}\text { List and define all variables for which data were sought (e.g. PICOS, funding sources) } \\
\text { and any assumptions and simplifications made. }\end{array}$ & $5-6$ \\
\hline $\begin{array}{l}\text { Risk of bias in } \\
\text { individual studies }\end{array}$ & 12 & $\begin{array}{l}\text { Describe methods used for assessing risk of bias of individual studies (including } \\
\text { specification of whether this was done at the study or outcome level), and how this } \\
\text { information is to be used in any data synthesis. }\end{array}$ & 6 \\
\hline Summary measures & 13 & State the principal summary measures (e.g. risk ratio, difference in means). & $6-7$ \\
\hline Synthesis of results & 14 & $\begin{array}{l}\text { Describe the methods of handling data and combining results of studies, if done, } \\
\text { including measures of consistency }\left(e . g . l^{2}\right) \text { for each meta-analysis. }\end{array}$ & $6-7$ \\
\hline $\begin{array}{l}\text { Risk of bias across } \\
\text { studies }\end{array}$ & 15 & $\begin{array}{l}\text { Specify any assessment of risk of bias that may affect the cumulative evidence (e.g. } \\
\text { publication bias, selective reporting within studies). }\end{array}$ & 7 \\
\hline Additional analyses & 16 & $\begin{array}{l}\text { Describe methods of additional analyses (e.g. sensitivity or subgroup analyses, } \\
\text { meta-regression), if done, indicating which were pre-specified. }\end{array}$ & $6-7$, Table 2 and Figure 3 \\
\hline \multicolumn{4}{|l|}{ RESULTS } \\
\hline Study selection & 17 & $\begin{array}{l}\text { Give numbers of studies screened, assessed for eligibility, and included in the review, } \\
\text { with reasons for exclusions at each stage, ideally with a flow diagram. }\end{array}$ & 7 \\
\hline Study characteristics & 18 & $\begin{array}{l}\text { For each study, present characteristics for which data were extracted (e.g. study size, } \\
\text { PICOS, follow-up period) and provide the citations. }\end{array}$ & 7-8, Table 1 \\
\hline $\begin{array}{l}\text { Risk of bias within } \\
\text { studies }\end{array}$ & 19 & $\begin{array}{l}\text { Present data on risk of bias of each study and, if available, any outcome level } \\
\text { assessment (see ltem 12). }\end{array}$ & 9, Figure 2 \\
\hline $\begin{array}{l}\text { Results of individual } \\
\text { studies }\end{array}$ & 20 & $\begin{array}{l}\text { For all outcomes considered (benefits or harms), present, for each study: (i) simple } \\
\text { summary data for each intervention group, (ii) effect estimates and confidence } \\
\text { intervals, ideally with a forest plot. }\end{array}$ & $9-10$ \\
\hline Synthesis of results & 21 & $\begin{array}{l}\text { Present results of each meta-analysis done, including confidence intervals and } \\
\text { measures of consistency. }\end{array}$ & 10 , Figure 3 \\
\hline $\begin{array}{l}\text { Risk of bias across } \\
\text { studies }\end{array}$ & 22 & Present results of any assessment of risk of bias across studies (see Item 15). & - \\
\hline Additional analysis & 23 & $\begin{array}{l}\text { Give results of additional analyses, if done (e.g. sensitivity or subgroup analyses, } \\
\text { meta-regression [see Item 16]). }\end{array}$ & 10, Table 2 \\
\hline \multicolumn{4}{|l|}{ DISCUSSION } \\
\hline Summary of evidence & 24 & $\begin{array}{l}\text { Summarize the main findings including the strength of evidence for each main outcome; } \\
\text { consider their relevance to key groups (e.g. healthcare providers, users and policy } \\
\text { makers). }\end{array}$ & 9 \\
\hline Limitations & 25 & $\begin{array}{l}\text { Discuss limitations at the study and outcome level (e.g. risk of bias), and at the review } \\
\text { level (e.g. incomplete retrieval of identified research, reporting bias). }\end{array}$ & $10-14$ \\
\hline Conclusions & 26 & $\begin{array}{l}\text { Provide a general interpretation of the results in the context of other evidence, and } \\
\text { implications for future research. }\end{array}$ & 14 \\
\hline \multicolumn{4}{|l|}{ FUNDING } \\
\hline Funding & 27 & $\begin{array}{l}\text { Describe sources of funding for the systematic review and other support (e.g. supply of } \\
\text { data); role of funders for the systematic review. }\end{array}$ & 15 \\
\hline
\end{tabular}

From Moher D, Liberati A, Tetzlaff J, Altman DG, The PRISMA Group (2009) Preferred Reporting Items for Systematic Reviews and Meta-Analyses: The PRISMA Statement. PLOS Med 6(7), e1000097. doi:10.1371/journal.pmed1000097. 
Pubmed

(('Morbid obesity'[All Fields] OR 'Morbid obesities'[All Fields] OR 'Severe obesity'[All Fields] OR ('obesity, morbid'[MeSH Terms] OR ('obesity'[All Fields] AND 'morbid'[All Fields]) OR 'morbid obesity'[All Fields] OR ('severe'[All Fields] AND 'obesities'[All Fields])) OR 'Abdominal obesities'[All Fields] OR 'Abdominal obesity'[All Fields] OR 'Central obesities'[All Fields] OR 'Central obesity'[All Fields] OR 'Visceral obesity'[All Fields] OR ('obesity, abdominal'[MeSH Terms] OR ('obesity'[All Fields] AND 'abdominal'[All Fields]) OR 'abdominal obesity'[All Fields] OR ('visceral'[All Fields] AND 'obesities'[All Fields])) OR 'Obese men'[All Fields] OR 'Obese women'[All Fields] OR 'Overweight'[All Fields] OR 'Overweight men'[All Fields] OR 'Overweight women'[All Fields] OR 'Excess weight'[All Fields] OR 'obese'[All Fields] OR 'obesity'[All Fields] OR 'Fat accumulation'[All Fields] OR 'fatness'[All Fields] OR 'body fatness'[All Fields]) AND ('N3 fatty acids'[All Fields] OR ' $n-3$ Fatty Acids'[All Fields] OR ' $n 3$ Fatty Acids'[All Fields] OR ' $n 3$ Fatty Acids'[All Fields] AND 'W3 fatty acids'[All Fields] OR 'w-3 fatty acids'[All Fields] OR ' $w 3$ fatty acids'[All Fields] OR 'N3 Polyunsaturated Fatty Acid'[All Fields] OR ' $n-3$ Polyunsaturated Fatty Acid'[All Fields] OR ' $n 3$ Polyunsaturated Fatty Acid'[All Fields] OR ' $n 3$ Polyunsaturated Fatty Acid'[All Fields] OR ' $n-3$ PUFA'[All Fields] OR ' $N 3$ PUFA'[All Fields] OR ' $N 3$ PUFA'[All Fields] OR ' $N-3$ oils'[All Fields] OR ('fatty acids, omega-3'[MeSH Terms] OR ('fatty'[All Fields] AND 'acids'[All Fields] AND 'omega-3'[All Fields]) OR 'omega-3 fatty acids'[All Fields] OR ('n3'[All Fields] AND 'oils'[All Fields])) OR ' $N 3$ oils'[All Fields] OR 'Omega 3 Fatty Acids'[All Fields] OR 'Eicosapentanoic Acid'[All Fields] OR 'omega 3 Eicosapentaenoic Acid'[All Fields] OR 'omega-3-Eicosapentaenoic Acid'[All Fields] OR 'Timnodonic Acid'[All Fields] OR 'Docosahexenoic Acid'[All Fields] OR (('fatty acids, omega-3'[MeSH Terms] OR ('fatty'[All Fields] AND 'acids'[All Fields] AND 'omega-3'[All Fields]) OR 'omega-3 fatty acids'[All Fields] OR 'omega 3'[All Fields]) AND Docosahexenoic[All Fields] AND ('acids'[MeSH Terms] OR 'acids'[All Fields] OR ‘acid'[All Fields])) OR ‘Docosahexaenoate'[All Fields] OR 'alpha Linolenic Acid'[All Fields] OR 'Linolenate'[All Fields] OR 'Linolenic Acid'[All Fields] OR 'EPA and DHA supplementation'[All Fields] OR EPA[All Fields] OR DHA[All Fields] OR 'omega 3'[All Fields] OR 'omega-3'[All Fields] OR 'fish oil'[All Fields] OR 'arachidonic acid'[All Fields] OR 'arachidonate'[All Fields] OR 'eicosatetraenoic acid'[All Fields])) AND (('eicosanoids'[MeSH Terms] OR 'eicosanoids'[All Fields] OR 'eicosanoid'[All Fields]) OR ('eicosanoids'[MeSH Terms] OR 'eicosanoids'[All Fields] OR 'icosanoid'[All Fields]) OR ('prostaglandins'[MeSH Terms] OR 'prostaglandins'[All Fields] OR 'prostanoid'[All Fields]) OR ('lipoxins'[MeSH Terms] OR 'lipoxins'[All Fields] OR 'lipoxin'[All Fields]) OR ('prostaglandins'[MeSH Terms] OR 'prostaglandins'[All Fields] OR 'prostaglandin'[All Fields]) OR ('thromboxanes'[MeSH Terms] OR 'thromboxanes'[All Fields] OR 'thromboxane'[All Fields]) OR ('leukotrienes'[MeSH Terms] OR 'leukotrienes'[All Fields] OR 'leukotriene'[All Fields]) OR 'hydroxyeicosatetraenoic acid'[All Fields] OR 'Isoprostane'[All Fields] OR 'dinoprostone'[All Fields])

Web of science

('Morbid obesity' OR 'Severe obesity' OR ‘Abdominal obesity' OR 'Central obesity' OR 'Visceral obesity' OR 'Obese men' OR 'Obese women' OR 'Overweight' OR 'Overweight men' OR 'Overweight women' OR 'Excess weight' OR 'obese' OR 'obesity' OR 'Fat accumulation' OR 'fatness' OR 'body fatness') AND TÓPICO: ('N3 fatty acids' OR ' $n-3$ Fatty Acids' OR ' $n 3$ Fatty Acids' OR ' $n 3$ Fatty Acids' 'W3 fatty acids' OR ' $w-3$ fatty acids' OR ' $w 3$ fatty acids' OR ' $N 3$ Polyunsaturated Fatty Acid' OR ' $n-3$ Polyunsaturated Fatty Acid' OR ' $n 3$ Polyunsaturated Fatty Acid' OR ' $n 3$ Polyunsaturated Fatty Acid' OR ' $n-3$ PUFA' OR ' $N 3$ PUFA' OR ' $N 3$ PUFA' OR ' $N-3$ oils' OR 'N3 oils' OR 'N 3 oils' OR 'Omega 3 Fatty Acids' OR 'Eicosapentanoic Acid' OR ‘omega 3 Eicosapentaenoic Acid' OR 'omega-3-Eicosapentaenoic Acid' OR 'Timnodonic Acid' OR 'Docosahexenoic Acid' OR 'omega 3 Docosahexenoic Acid' OR 'Docosahexaenoate' OR 'alpha Linolenic Acid' OR 'Linolenate' OR 'Linolenic Acid' OR 'EPA and DHA supplementation' OR EPA OR DHA OR 'omega 3' OR 'omega-3' OR 'fish oil' OR 'arachidonic acid' OR 'arachidonate' OR 'eicosatetraenoic acid') AND TÓPICO: (eicosanoid OR Icosanoid OR Prostanoid OR Lipoxin OR Prostaglandin OR Thromboxane OR Leukotriene OR 'hydroxyeicosatetraenoic acid' OR 'Isoprostane' OR 'dinoprostone')

Scopus TITLE-ABS-KEY (('Morbid obesity' OR 'Morbid obesities' OR 'Severe obesity' OR 'Severe obesities' OR 'Abdominal obesities' OR 'Abdominal obesity' OR 'Central obesities' OR 'Central obesity' OR 'Visceral obesity' OR 'Visceral obesities' OR 'Obese men' OR 'Obese women' OR 'Overweight' OR 'Overweight men' OR 'Overweight women' OR 'Excess weight' OR 'obese' OR 'obesity' OR 'Fat accumulation' OR 'fatness' OR 'body fatness' AND ' $N 3$ fatty acids' OR ' $n-3$ Fatty Acids' OR ' $n 3$ Fatty Acids' OR ' $n 3$ Fatty Acids' 'W3 fatty acids' OR ' $w-3$ fatty acids' OR ' $w 3$ fatty acids' OR 'N3 Polyunsaturated Fatty Acid' OR ' $n-3$ Polyunsaturated Fatty Acid' OR ' $n 3$ Polyunsaturated Fatty Acid' OR ' $n 3$ Polyunsaturated Fatty Acid' OR ' $n-3$ PUFA' OR ' $N 3$ PUFA' OR ' $N 3$ PUFA' OR ' $N-3$ oils' OR ' $N 3$ oils' OR ' $N 3$ oils' OR 'Omega 3 Fatty Acids' OR 'Eicosapentanoic Acid' OR 'omega 3 Eicosapentaenoic Acid' OR 'omega-3-Eicosapentaenoic Acid' OR 'Timnodonic Acid' OR 'Docosahexenoic Acid' OR 'omega 3 Docosahexenoic Acid' OR 'Docosahexaenoate' OR ‘alpha Linolenic Acid' OR 'Linolenate' OR 'Linolenic Acid' OR ‘EPA and DHA supplementation' OR epa OR dha OR 'omega 3' OR 'omega-3' OR 'fish oil' OR 'arachidonic acid' OR 'arachidonate' OR 'eicosatetraenoic acid' AND eicosanoid OR eicosanoid OR prostanoid OR lipoxin OR prostaglandin OR thromboxane OR leukotriene OR 'hydroxyeicosatetraenoic acid' OR 'Isoprostane' OR 'dinoprostone'))

Embase ('morbid obesity' OR 'severe obesity' OR 'abdominal obesity' OR 'central obesity' OR 'visceral obesity' OR 'obese men' OR 'obese women' OR 'overweight' OR 'overweight men' OR 'overweight women' OR 'excess weight' OR 'obese' OR 'obesity' OR 'fat accumulation' OR 'fatness' OR 'body fatness') AND ((' $n$-3 fatty acids' OR ' $n 3$ fatty acids' OR ' $n 3$ fatty acids') AND 'w3 fatty acids' OR ' $w-3$ fatty acids' OR ' $w 3$ fatty acids' OR ' $n-3$ polyunsaturated fatty acid' OR ' $n 3$ polyunsaturated fatty acid' OR ' $n 3$ polyunsaturated fatty acid' OR ' $n-3$ pufa' OR ' $n 3$ pufa' OR ' $n 3$ pufa' OR ' $n-3$ oils' OR ' $n 3$ oils' OR ' $n 3$ oils' OR 'omega 3 fatty acids' OR 'eicosapentanoic acid' OR 'omega 3 eicosapentaenoic acid' OR 'omega-3-eicosapentaenoic acid' OR 'timnodonic acid' OR 'docosahexenoic acid' OR 'omega 3 docosahexenoic acid' OR 'docosahexaenoate' OR 'alpha linolenic acid' OR 'linolenate' OR 'linolenic acid' OR 'epa and dha supplementation' OR epa OR dha OR 'omega 3' OR 'omega-3' OR 'fish oil' OR 'arachidonic acid' OR 'arachidonate' OR 'eicosatetraenoic acid') AND (eicosanoid OR icosanoid OR prostanoid OR lipoxin OR prostaglandin OR thromboxane OR leukotriene OR 'hydroxyeicosatetraenoic acid' OR 'isoprostane' OR 'dinoprostone')

Cochrane Title Abstract Keyword 'Morbid obesity' OR 'Severe obesity' OR 'Abdominal obesity' OR 'Central obesity' OR 'Visceral obesity' OR 'Obese men' OR 'Obese women' OR 'Overweight' OR 'Overweight men' OR 'Overweight women' OR 'Excess weight' OR 'obese' OR 'obesity' OR 'Fat accumulation' OR 'fatness' OR 'body fatness' in Title Abstract Keyword AND ' $N 3$ fatty acids' OR ' $n-3$ Fatty Acids' OR ' $n 3$ Fatty Acids' OR ' $n 3$ Fatty Acids' 'W3 fatty acids' OR ' $w-3$ fatty acids' OR ' $w 3$ fatty acids' OR ' $N 3$ Polyunsaturated Fatty Acid' OR ' $n-3$ Polyunsaturated Fatty Acid' OR ' $n 3$ Polyunsaturated Fatty Acid' OR ' $n 3$ Polyunsaturated Fatty Acid' OR ' $n-3$ PUFA' OR ' $N 3$ PUFA' OR ' $N 3$ PUFA' OR ' $N-3$ oils' OR ' $N 3$ oils' OR ' $N 3$ oils' OR 'Omega 3 Fatty Acids' OR 'Eicosapentanoic Acid' OR 'omega 3 Eicosapentaenoic Acid' OR 'omega-3-Eicosapentaenoic Acid' OR 'Timnodonic Acid' OR 'Docosahexenoic Acid' OR 'omega 3 Docosahexenoic Acid' OR 'Docosahexaenoate' OR ‘alpha Linolenic Acid' OR ‘Linolenate' OR 'Linolenic Acid' OR ‘EPA and DHA supplementation' OR EPA OR DHA OR 'omega 3' OR 'omega-3' OR 'fish oil' OR 'arachidonic acid' OR 'arachidonate' OR 'eicosatetraenoic acid' in Title Abstract Keyword AND eicosanoid OR Icosanoid OR Prostanoid OR Lipoxin OR Prostaglandin OR Thromboxane OR Leukotriene OR 'hydroxyeicosatetraenoic acid' OR 'Isoprostane' OR 'dinoprostone' in Title Abstract Keyword - (Word variations have been searched) 
Appendix 3. Excluded articles and reasons for exclusion (n 32)

Author, Year

Reason for exclusion

Aronson et al. (2011)

Allaire et al. (2016)

Denzlinger et al. (1995)

Djuric et al. (2017) $)^{(4)}$

Gammelmark et al. (2012)

Huerta et al. (2014)

Holt et al. (2017)

Lang et al. (2019) ${ }^{(3)}$

Murphy et al. (2007)

Newman et al. (2014)

Peres et al. (2018)

Petersson et al. (2010)

Pickens et al. (2015)

Shearer et al. (2018)

Trebble et al. (2004)

Young et al. (2011)

Celada et al. (2014)

Bohm et al. (2013)

Itariu et al. (2012)

Fisk et al. (2018)

Lengfelder et al. (2016)

Quach et al. (2017)

Uach et al. (2017)

Hill et al. (2007)

Gruslova et al. (2017)

Pickens et al. (2017) ${ }^{(2)}$

Qin et al. (2015)

Nieman et al. (2012)

Kaatz et al. (2004)

Garcia et al. (2016)

Garcia-Ravelo et al. (2018)

Brenner et al. (2017)

Stephensen et al. (2011)

Exclusion criteria were as follows: (1) absence of outcomes about nutritional status/ growth/quality of life; (2) qualitative study and (3) consensus/management/reviews/ letters/conference abstracts/editorials.

(1) Patients that underwent bariatric/metabolic surgery;

(2) Consensus, management, reviews, letters, conference abstracts, editorials;

(3) Patients with inflammatory diseases;

(4) Anti-inflammatory drugs or supplements; and

(5) Measurements of eicosanoids other than serum and plasma out of BMI range. 